\title{
DEEP ELECTRON TRAPS IN CdTe:In FILMS GROWN BY MOLECULAR BEAM EPITAXY*
}

\author{
A.K. Zakrzewski, L. Dobaczewski, G. Karczewski, T. Wojtowicz \\ AND J. KossuT \\ Institute of Physics, Polish Academy of Sciences \\ Al. Lotników 32/46, 02-668 Warszawa, Poland
}

\begin{abstract}
$N$-type indium doped CdTe grown on $n^{+}-\mathrm{GaAs}$ by molecular beam epitaxy has been studied by the standard deep level transient spectroscopy and the isothermal Laplace-transform deep level transient spectroscopy. It was found that the $\mathrm{Cd} / \mathrm{Te}$ flux ratio strongly influences the deep level transient spectroscopy results. The unusual temperature dependence of the electron emission rate in films grown at nearly stoichiometric conditions may point out that the observed defect is resonant with the conduction band.
\end{abstract}

PACS numbers: 68.55.Ln, 73.61.Ga

Any intentional doping of semiconductors may lead to a formation of undesired deep electronic states associated with point defects. The presence of deep states influences properties of the doped material and affects the performance of devices fabricated from it. Thus, an issue of primary importance is to develop such growth techniques which can eliminate deep states, or at least, which result in reducing their number in the material.

In two recent publications we have reported on the molecular beam epitaxy (MBE) growth and characterization of the $n$-type indium doped CdTe films grown on highly lattice mismatched GaAs substrates $[1,2]$. Here, we concentrate on the question how the MBE growth conditions, in particular, the deviation from the stoichiometric flux ratio, influences the creation of deep defect states in epitaxial $\mathrm{CdTe}$. The problems related to the formation of deep states accompanying in situ doping of MBE-grown CdTe remain insufficiently investigated, since except for Ref. [1] where we presented preliminary results of our deep level transient spectroscopy (DLTS) study, there are no other reports on this subject.

The Laplace transform analysis of capacitance transients and the standard deep level transient spectroscopy were employed to study the thermal emission of electrons from deep level defects in CdTe:In films. The DLTS spectra were taken by the SemiTrap DLS82E lock-in spectrometer, with the bias range from -1 to $-2 \mathrm{~V}$

*This work is supported by the State Committee for Scientific Research (Republic of Poland) through grants 8 T11B 02108 and 8 S501 03505. 
and the filling time of $0.05 \mathrm{~ms}$ under $0.1 \mathrm{~V}$. Capacitance transients of $0.4-400 \mathrm{~ms}$ were measured with $1 \mathrm{MHz}$ drive frequency.

A series of $n$-type indium doped CdTe films was grown on (100) $n^{+}-$GaAs substrates. For all the samples the $3.5 \mu \mathrm{m}$ thick topmost films (being the subject of the DLTS investigations) were grown at the same indium flux corresponding to the same In-cell temperature, $T_{\mathrm{In}}=550^{\circ} \mathrm{C}$, and at the same substrate temperature, $T_{\mathrm{s}}=250^{\circ} \mathrm{C}$. The films differ in the $\mathrm{Cd} / \mathrm{Te}$ flux ratio applied during their growth. Prior to the deposition of the topmost CdTe:In films, the GaAs substrate was covered with two buffer layers: $10 \AA$ of $\mathrm{ZnTe}$ and, subsequently, $3 \mu \mathrm{m}$ of CdTe heavily doped with In. The first layer was grown in order to reduce the lattice mismatch between $\mathrm{GaAs}$ and $\mathrm{CdTe}$, and the second, to eliminate the effect of compensation caused by dislocations in the vicinity of the $\mathrm{GaAs} / \mathrm{ZnTe} / \mathrm{CdTe}$ interface and thus, to ensure homogeneous doping of the topmost layer.

As the source material we used polycrystalline CdTe and an excess of elemental Cd or Te from separate cells. By using extra Cd or Te sources the $\mathrm{Cd} / \mathrm{Te}$ flux ratio was changed in a wide range, from 0.5 to 3.9 . It is worth noting that at strongly non-stoichiometric conditions, i.e., either for the strong excess of $\mathrm{Cd}$ $(\mathrm{Cd} / \mathrm{Te}>2)$ or $\mathrm{Te}$ overpressure $(\mathrm{Cd} / \mathrm{Te}<1)$, the reflection high energy electron diffraction (RHEED) pattern becomes spotty, which is a sign of the 3D growth mode. In agreement with earlier reports, at optimal conditions, i.e., at slight overpressure of $\mathrm{Cd}(1<\mathrm{Cd} / \mathrm{Te}<2)$, the growth proceeds in the $2 \mathrm{D}$ mode, which is manifested by pronounced diffraction streaks in the RHEED pattern.

From the analysis of $C-V$ characteristics we established that the net donor concentration $N_{\mathrm{D}}-N_{\mathrm{A}}$ in all our films was of the order of $10^{16} \mathrm{~cm}^{-3}$. Although the donor concentration was only slightly affected by the deviation from stoichiometry, the DLTS spectra changed dramatically. In the CdTe:In films grown at extremely $\mathrm{Cd}$ rich conditions $(\mathrm{Cd} / \mathrm{Te}>2$ ) we detected the presence of a thermal emission with a rate which exponentially depends on $10^{3} / T$. This dependence indicates that electrons are emitted from a single, well-defined deep state. The data yield following parameters of the state: the activation energy $E_{t}=0.59 \mathrm{eV}$ and the apparent capture cross section $\sigma_{n}=8 \times 10^{-14} \mathrm{~cm}^{2}$. On the other hand, the DLTS data obtained for films grown at nearly stoichiometric conditions $(\mathrm{Cd} / \mathrm{Te}=1.2 \div$ 1.4) are rather ambiguous. In this case, the DLTS detected a single emission rate too, but the temperature dependence of this emission rate is very unusual. With a decreasing rate window the DLTS peak shifts towards low temperatures following the exponential law, yielding the activation energy of $E_{t}=0.45 \mathrm{eV}$ in agreement with our preliminary results [1]. At low temperatures $(T<240 \mathrm{~K})$, however, the emission rate becomes nearly temperature independent. Because of this behavior, initially we interpreted this result as due to an appearance of a new emission peak with low activation energy (of the order of tenths of meV) and with an extremely low capture cross section (of the order of $10^{-18} \mathrm{~cm}^{2}$ ) [1].

In addition to the unusual temperature dependence, at low temperatures the DLTS peak becomes much broader than at $T>240 \mathrm{~K}$ which may suggest that the observed line is in fact a superposition of several processes with different emission rates. In order to verify the above hypothesis and, eventually, to separate constituents of the broad emission peak, we employed the Laplace DLTS - an 
experimental technique which applies the Laplace transform analysis to isothermal capacitance transients. The technique provides an order of magnitude better resolution than conventional DLTS [3]. Surprisingly, the high resolution Laplace DLTS did not reveal any fine structure for the electron emission process. As shown in Fig. 1 in the thermal emission process only one emission rate was revealed at all temperatures measured. Moreover, the Laplace DLTS data are in perfect agreement with the data derived from the standard DLTS.

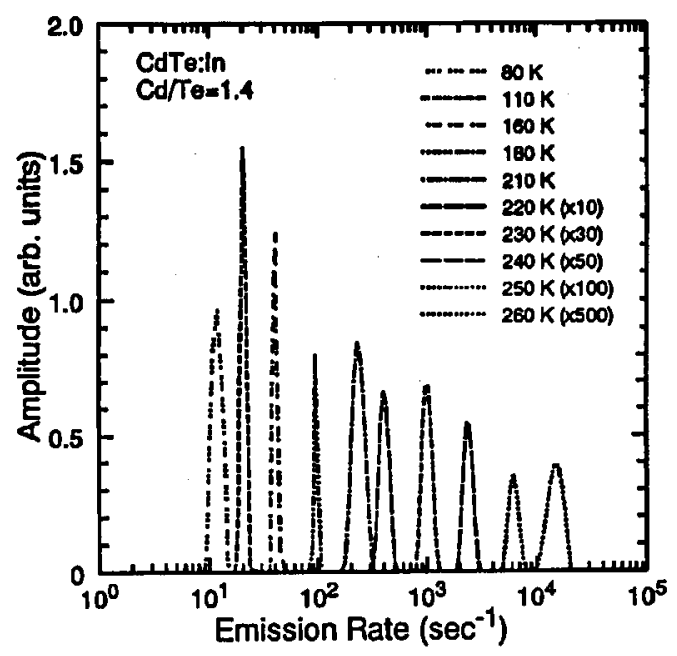

Fig. 1. Laplace DLTS spectra related measured on CdTe:In film at nearly stoichiometric flux conditions $(\mathrm{Cd} / \mathrm{Te}=1.4)$.

The temperature dependence of the thermal emission rate indicates that the emission of an electron from the deep state occurs via two competing processes. First is a thermally activated over-the-barrier electron transfer, the other one being a tunneling process. Such an effect can be observed only for defects which are resonant with the conduction band. Our DLTS results fully confirm results based on the Hall effect and resistivity measurements. They revealed that an In impurity in MBE-grown CdTe creates a localized resonant level laying about $125 \mathrm{meV}$ above the bottom of the conducting band $[4,5]$.

In summary, In-doped MBE-grown CdTe films were investigated using the standard and Laplace DLTS. Depending on the growth conditions, in particular, on the deviation from stoichiometry during the growth, two different electron traps have been observed. There are experimental evidences that the trap observed in films grown in nearly stoichiometric conditions is resonant with the conduction band.

The authors are thankful to Dr E. Kamińska and Dr A. Piotrowska, Institute of Electron Technology, Warsaw, for fabricating the Schottky diodes. 


\section{References}

[1] G. Karczewski, A.K. Zakrzewski, L. Dobaczewski, W. Dobrowolski, E. Grodzicka, J. Jaroszyński, T. Wojtowicz, J. Kossut, Thin Solid Films, in press.

[2] G. Karczewski, A.K. Zakrzewski, M. Kutrowski, J. Jaroszyński, W. Dobrowolski, E. Grodzicka, E. Janik, T. Wojtowicz, J. Kossut, A. Barcz, Acta Phys. Pol. A 87, 241 (1995).

[3] L. Dobaczewski, P. Kaczor, I.D. Hawkins, A.R. Peaker, J. Appl. Phys. 76, 194 (1994).

[4] D. Wasik, J. Przybytek, M. Baj, G. Karczewski, T. Wojtowicz, A. Zakrzewski, J. Kossut, Material Science Forum, in press.

[5] G.W. Iser, J.A. Kafalas, A.J. Strauss, Solid State Commun. 10, 619 (1972). 